Submitted to Physical Review C

FSU-SCRI-95-81

IU/NTC 95-10

$\mathrm{UK} / 95-08$

nucl-th/9508035

\title{
Isospin-Violating Meson-Nucleon Vertices as an Alternate Mechanism of Charge-Symmetry Breaking
}

\author{
S. Gardner \\ Department of Physics and Astronomy, \\ University of Kentucky, Lexington, KY 40506 \\ C. J. Horowitz \\ Nuclear Theory Center and Department of Physics, \\ Indiana University, Bloomington, IN 47505 \\ J. Piekarewicz \\ Supercomputer Computations Research Institute, \\ Florida State University, Tallahassee, FL 32306
}

(September 26, 2018)

\begin{abstract}
We compute isospin-violating meson-nucleon coupling constants and their consequent charge-symmetry-breaking nucleon-nucleon potentials. The couplings result from evaluating matrix elements of quark currents between nucleon states in a nonrelativistic constituent quark model; the isospin violations arise from the difference in the up and down constituent quark masses. We find, in particular, that isospin violation in the omega-meson-nucleon vertex dominates the class IV CSB potential obtained from these considerations. We evaluate the resulting spin-singlet-triplet mixing angles, the quantities germane to the difference of neutron and proton analyzing powers measured in elastic $\vec{n}-\vec{p}$ scattering, and find them commensurate to those computed originally using the on-shell value of the $\rho-\omega$ mixing amplitude. The use of the on-shell $\rho$ - $\omega$ mixing amplitude at $q^{2}=0$ has been called into question; rather, the amplitude is zero in a wide class of models. Our model possesses no contribution from $\rho$ - $\omega$ mixing at $q^{2}=0$, and we find that omega-meson exchange suffices to explain the measured $n-p$ analyzing power difference at $183 \mathrm{MeV}$.
\end{abstract}

PACS number(s): 11.30.-j, 21.30.+y 


\section{INTRODUCTION}

The suggestion of Goldman, Henderson, and Thomas [1] that the contribution of $\rho-\omega$ mixing to charge-symmetry-breaking (CSB) observables is suppressed in the low momentum transfer regime has opened the search for new sources of isospin violation. Since then, many calculations, using a variety of models, have confirmed the suppression of the $\rho$ $\omega$ mixing amplitude at small spacelike momenta [2 7]. Indeed, it has been shown that the $\rho$ - $\omega$ mixing amplitude is zero at $q^{2}=0$ in all models with vector mesons coupled to conserved currents [6]. Yet, in Refs. [2 7] no alternate mechanisms to $\rho-\omega$ mixing are proposed. The phenomenological impact of this gap must be emphasized: the CSB potential from $\rho-\omega$ mixing — with the mixing amplitude fixed at the omega-meson point — accounts for some $40 \%$ of the difference between the neutron and proton analyzing powers $(\Delta A)$ measured in elastic $\vec{n}-\vec{p}$ scattering at $183 \mathrm{MeV}$ [8]. Without this contribution the previous agreement between theory and experiment would be upset [9-[12]. Although the suppression of the mixing amplitude continues to be controversial [13, 14, sources of additional isospin violation are interesting in their own right and deserve examination. Indeed, the aim of the present paper is to show that a recently proposed CSB mechanism - based on isospinviolating meson-nucleon coupling constants [15] — is sufficient to restore the agreement with experiment. Specifically, we examine the effect of these new sources of CSB on the spin-singlet-triplet mixing angles; these are the fundamental dynamical quantities driving $\Delta A[10]$.

Most theoretical efforts devoted to understanding CSB observables use a nucleon-nucleon $(N N)$ interaction constrained by two-nucleon data [9, 10,16]. In such a picture, isospin violations arise from electromagnetic effects and hadronic mass differences. Sources of CSB can be classified in terms of three distinct contributions: (i) isovector-isoscalar mixing in the meson propagator, (ii) isospin-breaking in the nucleon wave function, and (iii) isospin breaking in the meson-nucleon and photon-nucleon vertices. Rho-omega mixing, the proton-neutron mass difference, and the difference between the electric charge of the proton and the neutron are typical examples of (i), (ii), and (iii), respectively. The existence of isovector-isoscalar mixing, such as $\pi-\eta$ and $\rho-\omega$ mixing, is well established. For example, $\rho-\omega$ mixing has been observed experimentally in $e^{+} e^{-} \rightarrow \pi^{+} \pi^{-}$measurements at the $\omega$-meson production point [17]. However, the suggested suppression of the mixing amplitudes at small spacelike momenta lessens their impact on CSB observables. This is, in part, why we consider other sources of isospin violation in this paper.

Isospin breaking in the nucleon wave function in a hadronic model is driven by the neutron-proton mass difference. Indeed, it is through this mechanism that charged-pion exchange dominates [18, 19] the class IV potential [20] at moderate momentum transfers. Isospin breaking in the nucleon wave function can also arise in a quark model picture from the mixing of the nucleon to $\left|J^{\pi}=1 / 2^{+} ; T=3 / 2\right\rangle$ baryon states [21]. While undoubtedly nonzero, one expects the $T=3 / 2$ components of the nucleon to be small due to the large mass difference between the nucleon and the $\Delta(1910)$ - the first $P_{31}$ baryon. In contrast, the $\rho-\omega$ mass difference is a mere $12 \mathrm{MeV}$. Thus, we turn to the meson-nucleon coupling constants as the possible sources of isospin violation demanded by data.

While there have been calculations of isospin-violating meson-nucleon coupling constants [11,22 25], their impact on class IV CSB observables has only recently been con- 
sidered [15]. Here, as earlier [15], we adopt a nonrelativistic quark model to calculate isospin breaking in the meson-nucleon coupling constants. In the model the coupling constants emerge from evaluating matrix elements of quark currents of the appropriate Lorentz and flavor structure between nucleon states. The isospin violations arise from the up-down quark mass difference. Radiative corrections to the vertices have also been evaluated and are found to be small [26,22]. Here we study the phenomenological impact of Ref. [15] in greater detail. In order to do this, we estimate the $q^{2}$ dependence of the isospin-breaking found in the vertices at $q^{2}=0$.

We have organized the paper as follows. In Sec. [1 the model is introduced, and isospinviolating meson-nucleon coupling constants are computed. We show that in the $q^{2}=0$ limit the couplings depend merely on the spin-flavor structure of the nucleon wave function; they are insensitive to the spatial components of the nucleon wave function. In Sec. [II] we use these findings to compute the resulting CSB potentials. In particular, we obtain a large contribution from omega-meson exchange to the class IV potential. We quantify the impact of isospin-violation in the $N N \omega$ vertex by computing the resulting spin-singlet-triplet mixing angles - these are the basic building blocks of $\Delta A$. These results are presented in Sec. $\mathbb{D V}$. Finally, we discuss the impact of our work in Sec. $\mathrm{D}$.

\section{ISOSPIN-VIOLATING MESON-NUCLEON COUPLING CONSTANTS}

We are interested in computing the coupling of an on-shell nucleon to the neutral mesons $\omega, \rho^{0}, \pi^{0}$, and $\sigma$. The off-shell vertices could engender additional isospin breaking, but our primary focus is on the $N N$ system, so that we will not consider these effects further. The exchanged mesons couple to nucleon currents of the appropriate Lorentz character, and the meson-nucleon coupling constants emerge from evaluating the matrix elements of these currents in the quark model. The difference in the up and down constituent quark masses thus gives rise to isospin-violating meson-nucleon coupling constants. At $q^{2}=0$ these couplings are determined from the spin and flavor structure of the nucleon wave function alone. In contrast, the couplings at $q^{2}=0$ of the nucleon to the charged mesons are sensitive to the quark momentum distribution as well, and are, therefore, more model dependent [11]. We shall consider the neutral-vector-meson-nucleon vertices first, as they are relevant to the $\Delta A$ measurement. The most general form for these on-shell $N N$-meson vertex functions, consistent with Lorentz covariance and parity invariance, are

$$
\begin{gathered}
-i g_{N N \omega} \Lambda_{N N \omega}^{\mu}=-i g_{N N \omega}\left(g_{N}^{\omega} \gamma^{\mu}+i f_{N}^{\omega} \sigma^{\mu \nu} \frac{\left(p^{\prime}-p\right)_{\nu}}{2 M_{N}}\right), \\
-i g_{N N \rho} \Lambda_{N N \rho}^{\mu}=-i g_{N N \rho}\left(g_{N}^{\rho} \gamma^{\mu}+i f_{N}^{\rho} \sigma^{\mu \nu} \frac{\left(p^{\prime}-p\right)_{\nu}}{2 M_{N}}\right),
\end{gathered}
$$

where $g_{N N \alpha}(\alpha=\omega, \rho)$ are the isospin-averaged, phenomenological, meson-nucleon coupling constants, determined from fits to the $N N$ phase shifts and to the properties of the deuteron [28,29], and $M_{N}$ is the nucleon mass. We compute the couplings, that is, $g_{N}^{\alpha}\left(q^{2}\right)$ and $f_{N}^{\alpha}\left(q^{2}\right)$, by assuming that the $N N \alpha$ vertex functions can be related to the matrix elements of quark currents of the appropriate Lorentz and flavor structure between nucleon states, computed in the nonrelativistic quark model. Thus, 


$$
\begin{aligned}
& \left\langle N\left(p^{\prime}, s^{\prime}\right)\left|J^{\mu ;+}\right| N(p, s)\right\rangle=\bar{U}\left(p^{\prime}, s^{\prime}\right) \Lambda_{N N \omega}^{\mu} U(p, s), \\
& \left\langle N\left(p^{\prime}, s^{\prime}\right)\left|J^{\mu ;-}\right| N(p, s)\right\rangle=\bar{U}\left(p^{\prime}, s^{\prime}\right) \Lambda_{N N \rho}^{\mu} U(p, s) .
\end{aligned}
$$

Here $U(p, s)$ denotes a on-shell nucleon spinor of mass $M_{N}$, momentum $p$, and spin $s$. We shall focus on the couplings at $q^{2}=0$, where $q \equiv p^{\prime}-p$, as the nonrelativistic quark model is best suited to an estimate in the static limit. The quark currents $J^{\mu ; \pm}$ are

$$
\begin{aligned}
J^{\mu ;+} & =\frac{1}{3} \bar{u} \gamma^{\mu} u+\frac{1}{3} \bar{d} \gamma^{\mu} d, \\
J^{\mu ;-} & =\bar{u} \gamma^{\mu} u-\bar{d} \gamma^{\mu} d .
\end{aligned}
$$

It is the quark vector current which is appropriate to the vector-meson-nucleon vertex; the second superscript $( \pm)$ denotes its symmetry under the $u \leftrightarrow d$ flavor transformation. Note that the constituent quarks are assumed to be elementary: no quark form factors have been introduced. The isoscalar vector quark charge is $1 / 3$, whereas the isovector vector quark charge is +1 for the up quark and -1 for the down quark. The charge assignments are made such that $g_{N}^{\omega}=1, g_{p}^{\rho}=1$, and $g_{n}^{\rho}=-1$, at $q^{2}=0$.

Our model stems from the notion of vector dominance [30. Vector dominance presumes that a photon's interaction with a nucleon is mediated by the rho — or omega - meson. Here we argue that the coupling of the vector mesons themselves to the nucleon can be determined via matrix elements of the appropriate isospin components of the quark vector current. Our model does not predict the isospin-conserving coupling constants $g_{N N \alpha}$; these must be extracted from phenomenological fits to two-nucleon data. However, the isospinviolating pieces, as well as the tensor-to-vector ratio, can be calculated within the model. Note that the vector dominance nature of our model implies that the quarks couple to conserved currents. We estimate the resulting coupling constants using the nonrelativistic quark model (NRQM); this is an additional assumption.

The couplings $g_{N}^{\alpha}$ and $f_{N}^{\alpha}$ are functions of the meson four-momentum $q^{2}$, though we shall focus on the couplings at $q^{2}=0$. In this limit the couplings are insensitive to the spatial component of the nucleon wave function; they follow directly from its spin and flavor content alone. In the $S U(6)$ limit 31,

$$
\begin{array}{r}
|p \uparrow\rangle=\frac{1}{\sqrt{18}}(2|u \uparrow u \uparrow d \downarrow\rangle-|u \uparrow u \downarrow d \uparrow\rangle-|u \downarrow u \uparrow d \uparrow\rangle+ \\
2|u \uparrow d \downarrow u \uparrow\rangle-|u \downarrow d \uparrow u \uparrow\rangle-|u \uparrow d \uparrow u \downarrow\rangle+ \\
2|d \downarrow u \uparrow u \uparrow\rangle-|d \uparrow u \uparrow u \downarrow\rangle-|d \uparrow u \downarrow u \uparrow\rangle) .
\end{array}
$$

The neutron spin-up wave function, $|n \uparrow\rangle$, is obtained by exchanging the up and down quarks in the expression for $|p \uparrow\rangle$. The isospin violations arise from the difference in the up and down constituent quark masses. The couplings constants are obtained from computing the matrix elements found in the nonrelativistic reduction of Eq. (2) in the quark model; i.e.,

$$
\begin{array}{lll}
g_{N}^{\omega}=\sum_{i=1}^{3} g_{i}^{+}\langle N \uparrow|1| N \uparrow\rangle & ; \quad & \frac{\left(g_{N}^{\omega}+f_{N}^{\omega}\right)}{2 M_{N}}=\sum_{i=1}^{3} \mu_{i}^{+}\left\langle N \uparrow\left|\sigma_{i}^{z}\right| N \uparrow\right\rangle, \\
g_{N}^{\rho}=\sum_{i=1}^{3} g_{i}^{-}\langle N \uparrow|1| N \uparrow\rangle & ; \quad \frac{\left(g_{N}^{\rho}+f_{N}^{\rho}\right)}{2 M_{N}}=\sum_{i=1}^{3} \mu_{i}^{-}\left\langle N \uparrow\left|\sigma_{i}^{z}\right| N \uparrow\right\rangle .
\end{array}
$$


Note that we have introduced the quark magnetic moment $\mu_{i}^{ \pm} \equiv g_{i}^{ \pm} / 2 m_{i}$, with the charges $g_{i}^{ \pm}$given in Table [. In the following presentation we discuss only the coupling of the nucleon to the $\omega$-meson, as an illustrative example. Our results, collected in Table II], include the couplings to the other mesons as well.

The vector coupling of the $\omega$-meson to the nucleon is determined by simply counting the quark charges:

$$
\begin{aligned}
& g_{p}^{\omega}=2 g_{u}^{+}+g_{d}^{+}=1, \\
& g_{n}^{\omega}=2 g_{d}^{+}+g_{u}^{+}=1 .
\end{aligned}
$$

The tensor coupling, in contrast, depends on the spin structure of the nucleon wave function:

$$
\begin{aligned}
& \mu_{p}^{\omega} \equiv \frac{g_{p}^{\omega}+f_{p}^{\omega}}{2 M_{p}}=\frac{4}{3} \mu_{u}^{+}-\frac{1}{3} \mu_{d}^{+}=\frac{1}{18}\left(\frac{4}{m_{u}}-\frac{1}{m_{d}}\right), \\
& \mu_{n}^{\omega} \equiv \frac{g_{n}^{\omega}+f_{n}^{\omega}}{2 M_{n}}=\frac{4}{3} \mu_{d}^{+}-\frac{1}{3} \mu_{u}^{+}=\frac{1}{18}\left(\frac{4}{m_{d}}-\frac{1}{m_{u}}\right) .
\end{aligned}
$$

It is useful to construct isoscalar and isovector combinations at the nucleon level; i.e.,

$$
\begin{aligned}
& g_{N}^{\omega}=g_{p}^{\omega} \frac{1}{2}\left(1+\tau_{z}\right)+g_{n}^{\omega} \frac{1}{2}\left(1-\tau_{z}\right) \equiv g_{0}^{\omega}+g_{1}^{\omega} \tau_{z}, \\
& \mu_{N}^{\omega}=\mu_{p}^{\omega} \frac{1}{2}\left(1+\tau_{z}\right)+\mu_{n}^{\omega} \frac{1}{2}\left(1-\tau_{z}\right) \equiv \mu_{0}^{\omega}+\mu_{1}^{\omega} \tau_{z},
\end{aligned}
$$

where

$$
\begin{aligned}
g_{0}^{\omega}+g_{1}^{\omega} \tau_{z} & =1 \\
\mu_{0}^{\omega}+\mu_{1}^{\omega} \tau_{z} & =\frac{1}{6 m}\left[1+\frac{5}{6} \frac{\Delta m}{m} \tau_{z}\right] \equiv\left[\frac{\left(g_{0}^{\omega}+f_{0}^{\omega}\right)}{2 M}+\frac{\left(g_{1}^{\omega}+f_{1}^{\omega}\right)}{2 M} \tau_{z}\right]
\end{aligned}
$$

with

$$
M \equiv \frac{1}{2}\left(M_{n}+M_{p}\right) ; \quad m \equiv \frac{1}{2}\left(m_{d}+m_{u}\right) ; \quad \Delta m \equiv\left(m_{d}-m_{u}\right) .
$$

The expression in Eq. (11) is given to leading order in $\Delta m / m$ only. Note that isospin breaking in the $f$ and $g$ couplings is realized in the $f$ alone and that the breaking in the $\omega$ tensor coupling is isovector in character. The $\omega$ - and $\rho$ - vector couplings are isospinconserving. The isospin-breaking in our model is connected to that of the electromagnetic form factors through our assumption of vector dominance; charge conservation protects the charge form factor from isospin-breaking at zero momentum transfer [21]. The tensor coupling is explicitly sensitive to the quark mass, as seen in Eq. (5), and the isospin-breaking corrections are generated by the up-down mass difference. In the constituent quark model $\Delta m>0$ [32]; the up quark, which is lighter, has a larger magnetic moment than the down quark. Henceforth we shall adopt the choice $M / 3 m \equiv 1$ in reporting the coupling constants.

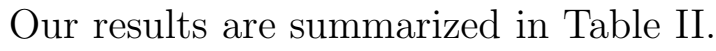

We now consider the isospin-conserving results. We find for the tensor-to-vector ratio that 


$$
\frac{f_{0}^{\omega}}{g_{N}^{\omega}}=0 ; \quad \frac{f_{1}^{\rho}}{g_{N}^{\rho}}=4 .
$$

These results are qualitatively consistent with the $f_{N}^{V} / g_{N}^{V}$ ratios which emerge from phenomenological fits to the $N N$ interaction [28,29] - recall that the Bonn B potential parameters [29], for example, are $f_{N}^{\omega} / g_{N}^{\omega}=0$ and $f_{N}^{\rho} / g_{N}^{\rho}=6.1$. This consistency is intimately connected to the NRQM's ability to describe the nucleon magnetic moments and to our assumption of vector dominance. In the NRQM, with $M=3 m$, the anomalous magnetic moment is purely isovector: $\kappa_{p}=2$ and $\kappa_{n}=-2$. Note that $\kappa_{p}^{\exp }=1.79$ and $\kappa_{n}^{\exp }=-1.91$. These successes gives us confidence in using our model to compute the isospin-violating corrections to these coupling constants.

For completeness, we shall now consider isospin breaking in the $N N \sigma$ and $N N \pi^{0}$ vertices as well. We exclude the $N N \eta$ vertex from this discussion because the $g_{N N \eta}$ coupling constant is poorly constrained by $N N$ data [33]. The appropriate vertex functions are

$$
\begin{aligned}
i g_{N N \sigma} \Lambda_{N N \sigma}^{\mathrm{s}} & =i g_{N N \sigma}\left(g_{N}^{\sigma}\right) 1, \\
g_{N N \pi} \Lambda_{N N \pi}^{5} & =g_{N N \pi}\left(g_{N}^{\pi}\right) \gamma^{5} .
\end{aligned}
$$

We have assumed pseudoscalar, rather than pseudovector, coupling for the pion in order to be consistent with earlier calculations of charge-symmetry breaking [9, 10, 16, 18, 19, In our model, we connect the vertex functions to matrix elements of quark currents, so that

$$
\begin{aligned}
& \left\langle N\left(p^{\prime}, s^{\prime}\right)\left|J^{\mathrm{s}}\right| N(p, s)\right\rangle=\bar{U}\left(p^{\prime}, s^{\prime}\right) \Lambda_{N N \sigma}^{\mathrm{s}} U(p, s) \\
& \left\langle N\left(p^{\prime}, s^{\prime}\right)\left|J^{5}\right| N(p, s)\right\rangle=\bar{U}\left(p^{\prime}, s^{\prime}\right) \Lambda_{N N \pi}^{5} U(p, s)
\end{aligned}
$$

where

$$
\begin{aligned}
& J^{\mathrm{s}}(q)=\frac{1}{3} \bar{u} u+\frac{1}{3} \bar{d} d, \\
& J^{5}(q)=\frac{1}{5} \bar{u} \gamma^{5} u-\frac{1}{5} \bar{d} \gamma^{5} d .
\end{aligned}
$$

The charges $g_{i}^{\mathrm{s}}=1 / 3, g_{u}^{5}=1 / 5$, and $g_{d}^{5}=-1 / 5$ have been chosen such that $g_{N}^{\sigma}=1, g_{p}^{\pi}=1$, and $g_{n}^{\pi}=-1$ when $\Delta m=0$. Evaluating the nonrelativistic reduction of Eqs. (15a) and (15) in the quark model, we find

$$
\begin{aligned}
g_{N}^{\sigma} & =\sum_{i=1}^{3} g_{i}^{\mathrm{s}}\langle N \uparrow|1| N \uparrow\rangle, \\
\frac{g_{N}^{\pi}}{2 M_{N}} & =\sum_{i=1}^{3} \mu_{i}^{5}\left\langle N \uparrow\left|\sigma_{i}^{z}\right| N \uparrow\right\rangle,
\end{aligned}
$$

where we have defined $\mu_{i}^{5} \equiv g_{i}^{5} / 2 m_{i}$. From Eq. (17a) we see that the sigma meson generates merely a spin-independent coupling to the nucleon in the nonrelativistic limit, so that there is no isospin breaking in the $N N \sigma$ vertex and no contribution from sigma exchange to the CSB potential. Thus, we will not consider sigma exchange further. However, the quark mass dependence contained in $\mu_{i}^{5}$ in Eq. (17b) implies that the isospin-breaking in the pion case is finite. The breaking to $\mathcal{O}(\Delta m / m)$ is indicated in Table $\mathbb{1}$. Note, however, that the 
computed breaking at $q^{2}=0$ depends on the nature of the assumed pion-nucleon coupling. If we had chosen pseudovector coupling, rather, then no isospin breaking would result. The pseudovector current contains no quark mass dependent pieces in the nonrelativistic limit. Thus, our prediction in the $\pi^{0}$ case is decidedly more model dependent than in the $\rho^{0}$ and $\omega$ channels. Moreover, in the latter case, the compatibility of the computed tensor-to-vector coupling constant ratios with the Bonn potential indicates that vector dominance, which we assume, has some phenomenological support. Note that in the $\pi^{0}$ case, there is no such independent support of our "pseudoscalar dominance" assumption. This concludes our discussion of isospin breaking in the $N N$-meson vertices.

\section{CHARGE SYMMETRY BREAKING POTENTIALS}

We shall now compute the CSB potentials which arise from the isospin-violating couplings computed in the previous section and tabulated in Table III. In an one-boson exchange approximation, presuming the form of the isospin breaking found in the $q^{2}=0$ results, we obtain the following CSB potentials for $\omega, \rho^{0}$, and $\pi^{0}$ exchange, respectively:

$$
\begin{aligned}
\widehat{V}_{\mathrm{CSB}}^{\omega} & =V_{\mathrm{CSB}}^{\omega}\left[\Gamma^{\mu}(1) \gamma_{\mu}(2) \tau_{z}(1)+\gamma^{\mu}(1) \Gamma_{\mu}(2) \tau_{z}(2)\right], \\
\widehat{V}_{\mathrm{CSB}}^{\rho} & =V_{\mathrm{CSB}}^{\rho} \Gamma^{\mu}(1) \Gamma_{\mu}(2)\left[\tau_{z}(1)+\tau_{z}(2)\right]+V_{\mathrm{CSB}}^{\rho}\left[\Gamma^{\mu}(1) \gamma_{\mu}(2) \tau_{z}(2)+\gamma^{\mu}(1) \Gamma_{\mu}(2) \tau_{z}(1)\right], \\
\widehat{V}_{\mathrm{CSB}}^{\pi} & =V_{\mathrm{CSB}}^{\pi} \gamma^{5}(1) \gamma^{5}(2)\left[\tau_{z}(1)+\tau_{z}(2)\right],
\end{aligned}
$$

where $\Gamma^{\mu} \equiv i \sigma^{\mu \nu}\left(p^{\prime}-p\right)_{\nu} / 2 M$ and we have defined

$$
\begin{gathered}
V_{\mathrm{CSB}}^{\omega}(q) \equiv-\left(\frac{g_{N N \omega}^{2}}{q^{2}-m_{\omega}^{2}}\right) f_{1}^{\omega} g_{0}^{\omega}, \\
V_{\mathrm{CSB}}^{\rho}(q) \equiv-\left(\frac{g_{N N \rho}^{2}}{q^{2}-m_{\rho}^{2}}\right) f_{0}^{\rho} g_{1}^{\rho}, \\
V_{\mathrm{CSB}}^{\prime \rho}(q) \equiv-\left(\frac{g_{N N \rho}^{2}}{q^{2}-m_{\rho}^{2}}\right) f_{0}^{\rho} f_{1}^{\rho}, \\
V_{\mathrm{CSB}}^{\pi}(q) \equiv-\left(\frac{g_{N N \pi}^{2}}{q^{2}-m_{\pi}^{2}}\right) g_{0}^{\pi} g_{1}^{\pi} .
\end{gathered}
$$

Isospin breaking in the meson-nucleon vertices give rise to the above CSB potentials, as per Eqs. (19a)-(19d). The isospin-conserving tensor coupling is nonzero in the case of the $\rho$ vertex, so that an additional potential of strength $V_{\mathrm{CSB}}^{\prime \rho}(q)$ arises. These contributions have been considered only recently [15]. Yet the potentials of Eqs. (18a) and (18d) are identical in form to those generated by $\rho-\omega$ and $\pi-\eta$ mixing, respectively. That is,

$$
\begin{aligned}
& \widehat{V}_{\mathrm{CSB}}^{\rho \omega}=V_{\mathrm{CSB}}^{\rho \omega}\left[\Gamma^{\mu}(1) \gamma_{\mu}(2) \tau_{z}(1)+\gamma^{\mu}(1) \Gamma_{\mu}(2) \tau_{z}(2)\right], \\
& \widehat{V}_{\mathrm{CSB}}^{\pi \eta}=V_{\mathrm{CSB}}^{\pi \eta} \gamma^{5}(1) \gamma^{5}(2)\left[\tau_{z}(1)+\tau_{z}(2)\right]
\end{aligned}
$$

where 


$$
\begin{aligned}
& V_{\mathrm{CSB}}^{\rho \omega}(q) \equiv-\frac{f_{N N \rho} g_{N N \omega}}{\left(q^{2}-m_{\rho}^{2}\right)\left(q^{2}-m_{\omega}^{2}\right)}\langle\rho|H| \omega\rangle, \\
& V_{\mathrm{CSB}}^{\pi \eta}(q) \equiv-\frac{g_{N N \pi} g_{N N \eta}}{\left(q^{2}-m_{\pi}^{2}\right)\left(q^{2}-m_{\eta}^{2}\right)}\langle\pi|H| \eta\rangle .
\end{aligned}
$$

Note that in Eq. (21a) we introduce $f_{N N \rho}$, the phenomenological tensor coupling of the Bonn model [29]. Rather than performing a nonrelativistic reduction of the potentials in Eqs. (18a)-(18c) and Eqs. (20a)-(20b), we simply classify the former as, either, " $\rho \omega-$ like" or " $\pi \eta$-like" potentials. The effect of these new isospin-violating potentials on CSB observables can then be readily elucidated. For example, the contribution from omega-meson exchange is identical in structure to that from $\rho$ - $\omega$ mixing and thus contributes as well to $\Delta A$ in elastic $\vec{n}-\vec{p}$ scattering. Indeed, we now show that the contribution from omega-meson exchange is comparable in magnitude and identical in sign to the one obtained from $\rho-\omega$ mixing - if the mixing amplitude is fixed at its on-shell value.

\section{One-boson exchange potentials of the $\rho$ - $\omega$ kind}

Potentials of the form given in Eq. (20a) give rise to class III and class IV CSB potentials. They are generated by the interference between the isospin-conserving vector coupling and the isospin-violating tensor coupling; note, for example, Eq. (18a) and the second term in Eq. (18b). Unlike the case of the omega, the isospin structure of rho exchange is not identical to that of $\rho$ - $\omega$ mixing; they are related by exchanging $\tau_{z}(1) \leftrightarrow \tau_{z}(2)$. Thus, rho exchange contributes to the class IV $\rho-\omega$ mixing potential with a sign opposite to that of the omega. No sign changes are necessary when computing its $\pi \eta$-like or class III $\rho \omega$-like contribution. Note that the contribution from rho exchange is small relative to that from the omega this emerges despite the larger isospin-violating coupling associated with the rho vertex (see Table [II). The vector $N N \rho$ coupling is simply small relative to that of the omega; in the Bonn potential $g_{N N \omega}^{2} / g_{N N \rho}^{2} \approx 27$ [29]. The relative importance of the various contributions can be estimated by computing the CSB potentials at $q^{2}=0$. Recall that in this limit the isospin-violating couplings are insensitive to the quark momentum distribution; they depend only on the spin-flavor symmetry of the wave function. Using the Bonn B potential parameters of Table [II] and a value for the quark-mass difference of $\Delta m=4.1 \mathrm{MeV}$ [32], we obtain the following results at $q^{2}=0$ :

$$
\begin{aligned}
& V_{\mathrm{CSB}}^{\omega}\left(q^{2}=0\right)=\frac{g_{N N \omega}^{2}}{m_{\omega}^{2}} f_{1}^{\omega} g_{0}^{\omega} \approx 2.49 \mathrm{GeV}^{-2}, \\
& V_{\mathrm{CSB}}^{\rho}\left(q^{2}=0\right)=\frac{g_{N N \rho}^{2}}{m_{\rho}^{2}} f_{0}^{\rho} g_{1}^{\rho} \approx 0.18 \mathrm{GeV}^{-2} . \\
& V_{\mathrm{CSB}}^{\rho \omega}\left(q^{2}=0\right)=-\left.\frac{f_{N N \rho} g_{N N \omega}}{m_{\rho}^{2} m_{\omega}^{2}}\langle\rho|H| \omega\rangle\right|_{q^{2}=0}=0,
\end{aligned}
$$

Several remarks are in order. First, the $\rho-\omega$ mixing amplitude, if modeled via fermion loops [2,6], necessarily vanishes at $q^{2}=0$ in our model. Our model assumes vector dominance, so that the vector-meson-nucleon vertices are determined by the appropriate isospin 
components of the quark electromagnetic current. Thus, the vector mesons couple to currents that are conserved at the nucleon level, so that the above result follows [2,6]. At the $q^{2}=0$ point, the charge-symmetry violation in our model comes purely from the vertex contributions. Note that the rho meson contribution to the latter is, indeed, small. It represents merely a $7 \%$ correction to the contribution from one-omega exchange. Second, the strength of the CSB potentials generated from omega exchange is comparable in magnitude to those obtained from $\rho-\omega$ mixing if the on-shell value of the mixing amplitude is assumed, $\left.\langle\rho|H| \omega\rangle\right|_{q^{2}=m_{\omega}^{2}}=-4520 \pm 600 \mathrm{MeV}^{2}$ [34]. Note, moreover, that the $\omega$ and on-shell $\rho-\omega$ mixing contributions are identical in sign. Specifically,

$$
\tilde{V}_{\mathrm{CSB}}^{\rho \omega}\left(q^{2}=0\right)=-\left.\frac{f_{N N \rho} g_{N N \omega}}{m_{\rho}^{2} m_{\omega}^{2}}\langle\rho|H| \omega\rangle\right|_{q^{2}=m_{\omega}^{2}} \approx 2.07 \mathrm{GeV}^{-2},
$$

A CSB potential of this magnitude is needed for a successful description of $\Delta A$ at 183 $\mathrm{MeV}$ [8]. Summing our omega exchange contribution to the CSB potential and that from on-shell $\rho-\omega$ mixing is not only internally inconsistent but also gives a final potential which

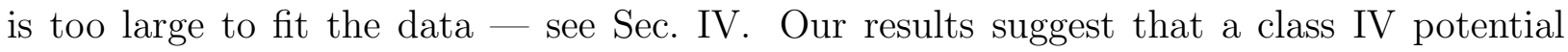
of the appropriate size is generated by isospin-violations in the $N N \omega$ vertex, together with small corrections from rho exchange and off-shell $\rho-\omega$ mixing. This is the central result of our paper.

\section{One-boson exchange potentials of the $\pi-\eta$ kind}

Potentials of the form given in Eq. (20b) generate class III CSB potentials exclusively. The Lorentz structure of the first term in Eq. (18b) differs from that of the $\pi-\eta$ mixing and one-pion exchange potentials, so that it is convenient to perform a nonrelativistic reduction of all three contributions, i.e.,

$$
\begin{aligned}
& \widehat{V}_{\mathrm{CSB}}^{\pi \eta}=-V_{\mathrm{CSB}}^{\pi \eta}(q)\left(\frac{\mathbf{q}^{2}}{12 M^{2}}\right)\left[\boldsymbol{\sigma}_{1} \cdot \boldsymbol{\sigma}_{2}+S_{12}(\hat{\mathbf{q}})\right]\left[\tau_{z}(1)+\tau_{z}(2)\right], \\
& \widehat{V}_{\mathrm{CSB}}^{\pi}=-V_{\mathrm{CSB}}^{\pi}(q)\left(\frac{\mathbf{q}^{2}}{12 M^{2}}\right)\left[\boldsymbol{\sigma}_{1} \cdot \boldsymbol{\sigma}_{2}+S_{12}(\hat{\mathbf{q}})\right]\left[\tau_{z}(1)+\tau_{z}(2)\right], \\
& \widehat{V}_{\mathrm{CSB}}^{\prime \rho}=-V_{\mathrm{CSB}}^{\prime \rho}(q)\left(\frac{\mathbf{q}^{2}}{12 M^{2}}\right)\left[2 \boldsymbol{\sigma}_{1} \cdot \boldsymbol{\sigma}_{2}-S_{12}(\hat{\mathbf{q}})\right]\left[\tau_{z}(1)+\tau_{z}(2)\right],
\end{aligned}
$$

where we have introduced the tensor operator $S_{12}(\hat{\mathbf{q}})=\left[3\left(\boldsymbol{\sigma}_{1} \cdot \hat{\mathbf{q}}\right)\left(\boldsymbol{\sigma}_{2} \cdot \hat{\mathbf{q}}\right)-\boldsymbol{\sigma}_{1} \cdot \boldsymbol{\sigma}_{2}\right]$. We estimate the relative size of these contributions by evaluating them at $q^{2}=0$, noting table एII:

$$
\begin{aligned}
& V_{\mathrm{CSB}}^{\pi}\left(q^{2}=0\right)=\frac{g_{N N \pi}^{2}}{m_{\pi}^{2}} g_{0}^{\pi} g_{1}^{\pi} \approx 36.83 \mathrm{GeV}^{-2} \\
& V_{\mathrm{CSB}}^{\prime \rho}\left(q^{2}=0\right)=\frac{g_{N N \rho} f_{N N \rho}}{m_{\rho}^{2}} f_{0}^{\rho} \approx 1.07 \mathrm{GeV}^{-2}
\end{aligned}
$$

The one-pion exchange contribution dominates that of the rho; this is driven by the large $\rho$ - $\pi$ mass difference - recall $m_{\rho}^{2} / m_{\pi}^{2} \approx 30$. Note that the inclusion of the rho meson leads 
to a reduction of the tensor and an enhancement of the spin-spin components of the pionexchange potential. Unlike the vector meson case, we cannot readily compute the $\pi-\eta$ mixing amplitude at $q^{2}=0$ in our model. That is, in the pion case, there is no conserved current, so that the $q^{2}=0$ mixing can be nonzero 35 37. Nevertheless, we can compare the results of Eq. (25) with the "usual" $\pi-\eta$ mixing potential:

$$
V_{\mathrm{CSB}}^{\pi \eta}\left(q^{2}=0\right)=-\left.\frac{g_{N N \pi} g_{N N \eta}}{m_{\pi}^{2} m_{\eta}^{2}}\langle\pi|H| \eta\rangle\right|_{q^{2}=m_{\eta}^{2}} \approx 52.01 \mathrm{GeV}^{-2}
$$

where we have input the $\pi-\eta$ mixing matrix element evaluated at its on-shell point, $\left.\langle\pi|H| \eta\rangle\right|_{q^{2}=m_{\eta}^{2}}=-4200 \mathrm{MeV}^{2}$ [33]. The contribution from one-pion exchange is comparable to that from $\pi-\eta$ mixing. The $\pi-\eta$ mixing potential may seem slightly larger, but the $N N \eta$ coupling is ill-determined from two-nucleon data. Indeed, it is believed that the Bonn potential overestimates it - a current analysis based on $\eta$-photoproduction data suggests couplings as low as $g_{N N \eta}^{2} / 4 \pi \lesssim 0.5$ [38] (see also Ref [35]).

The CSB potentials from one-pion exchange have been computed previously in a nucleon model [18]. Here the neutron-proton mass difference, $\Delta M$, generates the breaking. In the specific case of the class III contribution coming from neutral pion exchange, the scale of the breaking is set by $\Delta M / 2 M$. Thus, the isospin breaking in the quark model is substantially larger than in the nucleon model, i.e.,

$$
\left(\frac{3}{10} \frac{\Delta m}{m}\right) /\left(\frac{1}{2} \frac{\Delta M}{M}\right) \approx 6
$$

so that any CSB observable receiving an important contribution from $\pi-\eta$ mixing will also be affected by the exchange of neutral pions. The breaking we calculate in the $N N \pi^{0}$ vertex is identical to the result of Mitra and Ross [11,22]. Note that the exchange of charged pions and rhos - generates a class IV potential which is important in the analysis of $\Delta A$ [10,11]. In the charged meson case, however, the relation between the isospin-violating couplings in the two models is not simple: it depends on the quark momentum distribution. Yet, under reasonable assumptions, both sorts of models seem to generate class IV potentials of comparable strength [11].

\section{RESULTS}

In this section we compute the CSB potentials for a range of spacelike momenta. We shall concentrate on class IV contributions exclusively as we are interested in computing the impact of the new isospin-violating sources on $\Delta A$. The knowledge of the $q^{2}=0$ couplings now no longer suffices. One is forced to model the momentum dependence of the coupling constants - including that of the isospin-violating components. Here we consider two different estimates for the $q^{2}$ dependence of the CSB potentials. First, we simply adopt the momentum dependence which emerges from fits to the isospin-conserving two-nucleon data. Thus, the ratio of the isospin-violating to the isospin-conserving coupling, e.g., $f_{1}^{\omega} / g_{0}^{\omega}$, remains unchanged. Note that in the Bonn model $f_{1}^{\rho} / g_{1}^{\rho}$ is also a constant. We implement this choice by modifying the meson-nucleon "point" couplings indicated in Eq. (1) as per the Bonn B potential parameters, see Table [II]. That is, 


$$
\begin{gathered}
g_{N N \omega} \rightarrow g_{N N \omega}\left(\mathbf{q}^{2}\right)=g_{N N \omega}\left(1+\mathbf{q}^{2} / \Lambda_{\omega}^{2}\right)^{-2}, \\
g_{N N \rho} \rightarrow g_{N N \rho}\left(\mathbf{q}^{2}\right)=g_{N N \rho}\left(1+\mathbf{q}^{2} / \Lambda_{\rho}^{2}\right)^{-2} .
\end{gathered}
$$

This is an additional model assumption. Here we use $\mathbf{q}$ to denote the three-momentum transfer; we consider the form factors in the Breit frame, where $q_{0}=0$ and $q^{2}=-\mathbf{q}^{2}$. Second, we compute the $\mathcal{O}\left(\mathbf{q}^{2}\right)$ isospin-breaking in the couplings in the nonrelativistic quark model, in order to gauge the uncertainty in the momentum dependence of the CSB potentials. Let us examine the isospin-breaking in the Sachs-Walecka form factors [39], separated into contributions from the isoscalar or isovector quark charges. These quantities are related to the $\omega$ and $\rho$ couplings by virtue of our vector dominance assumption. As previously, we will discuss merely the isospin breaking in the $N N \omega$ vertex in detail. Now

$$
\begin{aligned}
G_{E, p}^{\omega} & =2 g_{u}^{+}\langle u\rangle_{p}+g_{d}^{+}\langle d\rangle_{p}, \\
G_{E, n}^{\omega} & =2 g_{d}^{+}\langle d\rangle_{n}+g_{u}^{+}\langle u\rangle_{n},
\end{aligned}
$$

and

$$
\begin{aligned}
\frac{G_{M, p}^{\omega}}{2 M_{p}} & =\frac{1}{18}\left(\frac{4}{m_{u}}\langle u\rangle_{p}-\frac{1}{m_{d}}\langle d\rangle_{p}\right), \\
\frac{G_{M, n}^{\omega}}{2 M_{n}} & =\frac{1}{18}\left(\frac{4}{m_{d}}\langle d\rangle_{n}-\frac{1}{m_{u}}\langle u\rangle_{n}\right) .
\end{aligned}
$$

These expressions are generalizations of Eqs. (6) and (7). We have used the notation of Eq. (50) in denoting the isoscalar and isovector quark charges and have introduced $\langle u\rangle_{p}$, for example, to represent the Fourier transform of the proton wave function with respect to the up quark coordinate. We compute the latter in the harmonic oscillator quark model for simplicity. In the harmonic oscillator quark model [21,40] the nucleon possesses a mass $M_{N}=2 m_{1}+m_{2}$, so that for the proton $m_{1}=m_{u}$ and $m_{2}=m_{d}$. For convenience one defines $R_{\rho}^{-2}=\sqrt{3 k m_{\rho}}$ and $R_{\lambda}^{-2}=\sqrt{3 k m_{\lambda}}$, where $m_{\lambda}=3 m_{1} m_{2} /\left(2 m_{1}+m_{2}\right), m_{\rho}=m_{1}$, and $k$ is the spring constant. One finds that 21]

$$
\begin{aligned}
\langle u\rangle_{p} & \equiv\left\langle\exp \left(i \mathbf{q} \cdot \mathbf{r}_{u}\right)\right\rangle_{p}=1-\frac{\mathbf{q}^{2}}{8}\left(R_{\rho p}^{2}+3\left(\frac{m_{d}}{M_{p}}\right)^{2} R_{\lambda p}^{2}\right)+\mathcal{O}\left(\mathbf{q}^{4}\right), \\
\langle d\rangle_{p} & \equiv\left\langle\exp \left(i \mathbf{q} \cdot \mathbf{r}_{d}\right)\right\rangle_{p}=1-\frac{3 \mathbf{q}^{2}}{2}\left(\frac{m_{u}}{M_{p}}\right)^{2} R_{\lambda p}^{2}+\mathcal{O}\left(\mathbf{q}^{4}\right), \\
\langle u\rangle_{n} & \equiv\left\langle\exp \left(i \mathbf{q} \cdot \mathbf{r}_{u}\right)\right\rangle_{n}=1-\frac{3 \mathbf{q}^{2}}{2}\left(\frac{m_{d}}{M_{n}}\right)^{2} R_{\lambda n}^{2}+\mathcal{O}\left(\mathbf{q}^{4}\right), \\
\langle d\rangle_{n} & \equiv\left\langle\exp \left(i \mathbf{q} \cdot \mathbf{r}_{d}\right)\right\rangle_{n}=1-\frac{\mathbf{q}^{2}}{8}\left(R_{\rho n}^{2}+3\left(\frac{m_{u}}{M_{n}}\right)^{2} R_{\lambda n}^{2}\right)+\mathcal{O}\left(\mathbf{q}^{4}\right) .
\end{aligned}
$$

We write the Fourier transforms in Eq. (31) through $\mathcal{O}\left(\mathbf{q}^{2}\right)$ only. This suffices to make contact with the hadronic form factors. Moreover, one cannot expect the nonrelativistic quark model to be reliable at still larger momentum transfers. We must now relate the above electric and magnetic form factors to the $f$ 's and $g$ 's present in the definition of the vertex, Eq. (11). Following the usual relation between the electromagnetic form factors $G_{E}$, $G_{M}$ and $F_{1}, F_{2}$, vector dominance dictates that 


$$
\begin{aligned}
& G_{E, N}^{\omega}\left(q^{2}\right)=g_{N}^{\omega}\left(q^{2}\right)+\frac{q^{2}}{4 M_{N}^{2}} f_{N}^{\omega}\left(q^{2}\right), \\
& G_{M, N}^{\omega}\left(q^{2}\right)=g_{N}^{\omega}\left(q^{2}\right)+f_{N}^{\omega}\left(q^{2}\right) .
\end{aligned}
$$

Fits to the electromagnetic form factor data indicate that $F_{1}\left(q^{2}\right)$ and $F_{2}\left(q^{2}\right)$ fall with different rates in $q^{2}$; vector dominance implies that this should be true of $g_{N}^{\omega, \rho}$ and $f_{N}^{\omega, \rho}$ as well. Note, this is at odds with the Bonn model, as it assumes that the ratio $f_{N}^{\omega, \rho} / g_{N}^{\omega, \rho}$ is constant. We proceed as follows. We compute $f_{N}^{\omega}$ and $g_{N}^{\omega}$ to $\mathcal{O}\left(\mathbf{q}^{4}, \Delta m^{2}\right)$, using Eqs. (29) 32). Then we estimate the "effective" $\Lambda_{\omega}$, as defined in Eq. (28), required to reproduce the isospin breaking computed to $\mathcal{O}\left(\mathbf{q}^{4}, \Delta m^{2}\right)$ and use that $\Lambda_{\omega}$ in our subsequent computation of the spin-singlet-triplet mixing angles. Thus,

$$
\begin{aligned}
g_{0}^{\omega}+g_{1}^{\omega} \tau_{z} & =\left[1-\frac{\mathbf{q}^{2} R^{2}}{6}\right]+\frac{\Delta m}{m}\left[\frac{5 \mathbf{q}^{2}}{24 M^{2}}-\frac{\mathbf{q}^{2} R^{2}}{72}\right] \tau_{z}+\mathcal{O}\left(\mathbf{q}^{4}, \Delta m^{2}\right), \\
f_{0}^{\omega}+f_{1}^{\omega} \tau_{z} & =\frac{5}{6} \frac{\Delta m}{m}\left[1-\frac{\mathbf{q}^{2} R^{2}}{3}-\frac{\mathbf{q}^{2}}{4 M^{2}}\right] \tau_{z}+\mathcal{O}\left(\mathbf{q}^{4}, \Delta m^{2}\right) .
\end{aligned}
$$

Several remarks are in order. First, note that we have defined $R^{-2}=\sqrt{3 k m}$, where $m$ is the average mass of the up and down quarks. From Eq. (33b) we observe $f_{0}^{\omega}=0$ to $\mathcal{O}\left(\mathbf{q}^{4}, \Delta m^{2}\right)$; this is consistent with the Bonn model, which assumes $f_{0}^{\omega}=0$ for all $q^{2}$. We have performed the same calculations for the $N N \rho$ vertex as well. In this case, one finds results at odds with the Bonn model, as $f_{1}^{\rho} / g_{1}^{\rho}$ is not constant to $\mathcal{O}\left(\mathbf{q}^{4}, \Delta m^{2}\right)$. Note that at nonzero $\mathbf{q}^{2}$ CSB potentials beyond those enumerated in Eqs. (19a)-(19d) may exist. For example, at $\mathcal{O}\left(\mathbf{q}^{2}, \Delta m\right)$ a new CSB contribution arises from the combination $f_{1}^{\rho} g_{0}^{\rho}$. Yet, like the rho contribution to the CSB potential given in Eq. (19b), it is not numerically important, due to the small value of $g_{N N \rho}$ in the Bonn model - recall that $g_{N N \omega}^{2} / g_{N N \rho}^{2} \approx 27$ [29]. Let us proceed to examine the impact of Eq. (33B) on the omega contribution to the class IV CSB potential. We fix the scale $R$ by requiring that the isospin conserving vertex, $g_{0}^{\omega}$, fall in $\mathbf{q}^{2}$ at the rate given by the Bonn model, so that $R=\sqrt{12} / \Lambda_{\omega} \approx .37 \mathrm{fm}$. We choose $R$ in this manner as our primary interest is in determining the fall-off of the isospin-breaking potential relative to the isospin-conserving one. Noting Eq. (19a), we consider

$$
\begin{aligned}
f_{1}^{\omega} g_{0}^{\omega} & =\frac{5}{6} \frac{\Delta m}{m}\left(1-6 \frac{\mathbf{q}^{2}}{\Lambda_{\omega}^{2}}-\frac{\mathbf{q}^{2}}{4 M^{2}}\right)+\mathcal{O}\left(\mathbf{q}^{4}, \Delta m^{2}\right) \\
& \equiv \frac{5}{6} \frac{\Delta m}{m}\left(1-4 \frac{\mathbf{q}^{2}}{\widetilde{\Lambda}_{\omega}^{2}}+\mathcal{O}\left(\mathbf{q}^{4}\right)\right) .
\end{aligned}
$$

By replacing the $\Lambda_{\omega}$ of Eq. (28) with the $\widetilde{\Lambda}_{\omega}$ given above, such that

$$
\widetilde{\Lambda}_{\omega}^{2}=\Lambda_{\omega}^{2}\left(\frac{4}{6+\Lambda_{\omega}^{2} / 4 M^{2}}\right),
$$

we obtain an expression for the CSB potential, Eq. (19a), which is of the form given by our original prescription, Eq. (28), yet is equivalent to the isospin-breaking calculated in the harmonic oscillator quark model at $\mathcal{O}\left(\mathbf{q}^{2}, \Delta m\right)$. Numerically, the Bonn model $\Lambda_{\omega}=1850$ $\mathrm{MeV}$ is changed to $\widetilde{\Lambda}_{\omega}=1401 \mathrm{MeV}$. At this order the coefficient of $g_{1}^{\rho}$ is not negative, so 
that we cannot carry out the above exercise for the rho as well. The rho's numerical impact on the CSB potential is small, so that this gap does not impact our uncertainty estimate in any significant way. We will proceed to compute the spin-singlet-triplet mixing angles for the potential given by Eqs. (19a) and (28) for both the $\Lambda_{\omega}$ of the Bonn potential and the $\widetilde{\Lambda}_{\omega}$ of Eq. (35).

In Fig. [1 we present estimates of the CSB potentials given in Eqs. (19a), (19b), and (21a) using Eq. (28) with the $\Lambda_{\omega, \rho}$ of the Bonn model. The qualitative conclusions we draw here are not sensitive to the choice of $\Lambda_{\omega, \rho}$, so that we simply present the potentials computed in the Bonn model. The solid line is the CSB potential which results from $\rho-\omega$ mixing, Eq. (21a), if the on-shell value of the $\rho-\omega$ mixing amplitude is employed for the entire range of momenta. This is the potential traditionally used in studies of CSB observables. A potential of this strength is required to describe the analyzing power difference $\Delta A$ measured in elastic $\vec{n}-\vec{p}$ scattering [16]. In contrast, the dashed line results if the momentum-dependent $\rho-\omega$ mixing amplitude of Ref. [2] is employed in Eq. (21a) - this is too small to fit the data [12], yet a model in which the vector mesons couple to conserved currents must yield a vanishing mixing amplitude at $q^{2}=0$ [6]. We have not extended our model to describe $\rho$ - $\omega$ mixing; the vector dominance assumption we use implies, however, that the $q^{2}=0$ mixing must be zero in this framework. We take the momentum-dependence of the mixing amplitude computed by Piekarewicz and Williams [2] as archetypal. This latter CSB potential in itself would upset the previous agreement with experiment. However, the new sources of isospin violation computed here are sufficient to restore the agreement. In particular, the contribution from omega-meson exchange, given by the dash-dotted line, is large and comparable in magnitude to the one arising from on-shell $\rho-\omega$ mixing. We have also computed the contribution from the rho-meson, given by the dotted line, though it is negligible due to the small $N N \rho$ vector coupling.

In Fig. 2 we display the above CSB potentials in configuration space. The potentials have been normalized so that the areas under the curves equal $V\left(q^{2}=0\right)$. Qualitatively, the trends observed in Fig. 1 1 remain: we obtain large contributions from on-shell $\rho$ - $\omega$ mixing and omega-meson exchange and small corrections to the latter from off-shell $\rho-\omega$ mixing and rho-meson exchange. These results are suggestive, yet we can obtain a precise estimate of the impact of the enumerated isospin-violating sources on $\Delta A$ by calculating the spin-singlettriplet mixing angles, $\bar{\gamma}_{J}$. These are the dynamical quantities driving $\Delta A$ 10,11. Recall that the elastic scattering amplitude of two spin-1/2 particles is specified by six invariant amplitudes $a, b, c, d, e$, and $f$ [11], so that

$$
\begin{aligned}
\widehat{M} & =\frac{1}{2}\left[(a+b)+(a-b)\left(\sigma_{1} \cdot \hat{\mathbf{n}}\right)\left(\sigma_{2} \cdot \hat{\mathbf{n}}\right)\right. \\
& +(c+d)\left(\sigma_{1} \cdot \hat{\mathbf{m}}\right)\left(\sigma_{2} \cdot \hat{\mathbf{m}}\right)+(c-d)\left(\sigma_{1} \cdot \hat{\mathbf{l}}\right)\left(\sigma_{2} \cdot \hat{\mathbf{l}}\right) \\
& \left.+e\left(\sigma_{1}+\sigma_{2}\right) \cdot \hat{\mathbf{n}}+f\left(\sigma_{1}-\sigma_{2}\right) \cdot \hat{\mathbf{n}}\right],
\end{aligned}
$$

where

$$
\hat{\mathbf{l}} \equiv \frac{\mathbf{k}_{f}+\mathbf{k}_{i}}{\left|\mathbf{k}_{f}+\mathbf{k}_{i}\right|}, \quad \hat{\mathbf{m}} \equiv \frac{\mathbf{k}_{f}-\mathbf{k}_{i}}{\left|\mathbf{k}_{f}-\mathbf{k}_{i}\right|}, \quad \hat{\mathbf{n}} \equiv \frac{\mathbf{k}_{i} \times \mathbf{k}_{f}}{\left|\mathbf{k}_{i} \times \mathbf{k}_{f}\right|},
$$

and $\mathbf{k}_{i}$ and $\mathbf{k}_{f}$ are the initial and final c.m. momenta of particle 1 . The $\vec{n}-\vec{p}$ analyzing power difference is nonzero only if accompanied by spin-singlet-triplet mixing, specifically 


$$
\Delta A(\theta) \equiv A_{n}(\theta)-A_{p}(\theta)=2 \operatorname{Re}\left(b^{*} f\right) / \sigma_{0},
$$

where $\sigma_{0}$ is the unpolarized differential cross section. The spin-singlet-triplet mixing is controlled by $f$. Neglecting electromagnetic effects, $f$ is connected to the mixing angles $\bar{\gamma}_{J}$ via 19

$$
f(k, \theta)=\frac{i}{2 k} \sum_{J=1}^{\infty}(2 J+1) \sin \left(2 \bar{\gamma}_{J}\right) \exp \left(i \bar{\delta}_{J}+i \bar{\delta}_{J J}\right) d_{10}^{J}(\theta),
$$

where the $d_{10}^{J}(\theta)$ are Wigner functions and the $\bar{\delta}_{J}$ and $\bar{\delta}_{J J}$ are the singlet and uncoupled triplet bar phase shifts, respectively. In a distorted-wave Born approximation the mixing angles themselves are given by [10]

$$
\bar{\gamma}_{J}=-4 M k \sqrt{J(J+1)} \int_{0}^{\infty} d r r^{2} R_{J}(r) V_{I V}(r) R_{J J}(r) \equiv \int_{0}^{\infty} d r I_{J}(r),
$$

where we have introduced the class IV CSB potential

$$
V_{I V}(r) \equiv \frac{1}{2 M^{2} r} \frac{d V_{\mathrm{CSB}}(r)}{d r} .
$$

Note that $R_{J}(r)$ and $R_{J J}(r)$ are the spin-singlet and triplet radial wave functions, respectively, for $N N$ scattering in the $L=J$ channel. Distortion effects are incorporated through these radial wave functions; we assume them adequately described by solutions to the Reid soft-core potential [4]. In Table $\mathbb{1 V}$ the first four nonvanishing mixing angles, $J=1-4$, are presented at a laboratory energy of $183 \mathrm{MeV}$. In addition, the integrand from which $\bar{\gamma}_{1}$ is obtained, that is, $I_{1}(r)$ in Eq. (40), is plotted in Fig. 3. This represents the class IV potential suitably weighted by realistic $N N$ wave functions. Three calculations are presented for comparison. The solid line is obtained using Eq. (21a) and the on-shell value of the $\rho-\omega$ mixing amplitude; the area under this curve is the mixing angle required to reproduce the $\Delta A$ data. In the dashed line we have combined the off-shell $\rho-\omega$ mixing contribution described above with the isospin-violating vertex contributions arising from omega- and rho-meson exchange. Albeit form factor uncertainties in the isospin-violating vertices, this is our best estimate of the mixing angle contribution. The vertices in this figure were evaluated using Eq. (28) and the Bonn cutoff parameters $\Lambda_{\omega, \rho}$ tabulated in Table III. We have also combined the on-shell $\rho-\omega$ mixing contribution with the above vertex contributions, even if our model is not consistent with a nonzero mixing amplitude at $q^{2}=0$ - this is shown by the dash-dotted line. The integrand in this case is considerably larger than the other two estimates. The $J=1$ mixing angles for these integrands are displayed in parentheses next to the curve labels. The agreement between the first two calculations is very good. Indeed, the contribution from omega-meson exchange, together with small corrections from off-shell $\rho-\omega$ mixing and rho-meson exchange, results in a $3 \%$ reduction in the value of $\bar{\gamma}_{1}$, relative to the on-shell value. This kind of agreement - at the few percent level - is maintained throughout all the examined partial waves, note Table $\mathbb{T}$. These computations have also been performed with the form factor $\widetilde{\Lambda}_{\omega}$, Eq. (35), estimated in the harmonic oscillator quark model. The mixing angles obtained in this fashion vary by about $10 \%$ in the important partial waves from those computed with the Bonn form factors; note that $\bar{\gamma}_{1}=.036^{\circ}$, rather than $.033^{\circ}$. 
For a detailed comparison, see Table IV - the mixing angles which use the harmonic oscillator quark model results to estimate the $\mathbf{q}^{2}$ dependence of the CSB potentials are shown in parentheses. The mixing angles computed with isospin-breaking meson-nucleon vertices and off-shell $\rho$ - $\omega$ mixing in the two approaches bracket the old on-shell $\rho-\omega$ mixing results for $J=1-3$, so that these new estimates are also quite close to the "old" on-shell results. $\widetilde{\Lambda}_{\omega}$ is some $3 / 4$ of $\Lambda_{\omega}$, yet the above calculations show that the $\Delta A$ at $183 \mathrm{MeV}$ is essentially dominated by the $q^{2}=0$ physics. Note that if one were to assume a momentum-independent $\rho$ - $\omega$ mixing amplitude [13 and to include the contributions from omega and rho exchange an increase of almost a factor of two relative to the above mixing angle estimates would result.

\section{CONCLUSIONS}

We have studied the charge-symmetry breaking in the $N N$ potential arising from isospinviolating meson-nucleon coupling constants. The isospin-violating couplings are obtained by computing matrix elements of quark currents of the appropriate Lorentz and flavor structure between nucleon states. We have used a nonrelativistic quark model to evaluate these matrix elements, yet our estimates at $q^{2}=0$ depend merely on the spin and flavor structure of the nucleon wave function, rather than on the details of the quark momentum distribution. Thus, in the vector meson sector, for example, our model estimates at $q^{2}=0$ depend on our vector dominance assumption, but little else. We have also studied isospin breaking in the $N N \pi$ and $N N \sigma$ vertices. No isospin-violations exist in the $\sigma$ vertex at $q^{2}=0$. We have found that the breaking in the $N N \pi$ vertex depends on whether the $\pi \mathrm{N}$ coupling is of pseudoscalar or pseudovector character - no isospin breaking results if pseudovector coupling is assumed. However, a pseudoscalar $\pi \mathrm{N}$ coupling is commonly used in studies of CSB, and the breaking we find in the vertex is substantially larger than the breaking computed in hadronic models of neutral pion exchange. Thus, any CSB observable receiving an important contribution from $\pi-\eta$ mixing will also be affected by the exchange of neutral pions.

We have found that omega-meson exchange is an important component of the class IV charge-symmetry-breaking $N N$ potential needed to describe the analyzing power difference measured in elastic $\vec{n}-\vec{p}$ scattering at low energies. The potential which emerges from the isospin-violating $N N \omega$ vertex is identical in structure to that from $\rho-\omega$ mixing [15. Moreover, our estimates indicate that these two contributions - with the mixing amplitude fixed at its on-shell value - are comparable in magnitude and identical in sign at $q^{2}=0$. Models in which the vector mesons couple to conserved currents, of which ours is an example, have no $\rho$ - $\omega$ mixing at $q^{2}=0$ [6]. We have found that isospin-violation in the $N N \omega$ vertex can generate a CSB potential of sufficient magnitude to fill the phenomenological role required by the IUCF measurement of $\Delta A$ at $183 \mathrm{MeV}$.

The isospin-violating couplings we have computed at $q^{2}=0$ do not suffice to make a quantitative prediction of the CSB potential needed for the IUCF experiment. One must compute the $q^{2}$ dependence of the $N N$ meson vertex as well - including that of the isospinviolating pieces. We have considered two simple estimates. The first is simply a prescription: we modify the "point" couplings by introducing hadronic form factors according to the Bonn $B$ potential. This assumes that the relative strength of the isospin-breaking potential found at $q^{2}=0$ persists at finite $q^{2}$ as well. In the second we compute the isospin breaking to 
$\mathcal{O}\left(\mathbf{q}^{4}, \Delta m^{2}\right)$ using the spatial wave functions of the harmonic oscillator quark model and find the hadronic form factor for omega exchange needed to reproduce the isospin breaking computed to the above order. The use of the spatial component of the nucleon wave function is required here, so that this estimate is rather more model dependent than our $q^{2}=0$ results. We find that the use of the latter estimate yields slightly larger CSB potentials.

Armed with estimates of the momentum dependence of the $N N$ meson vertex, we have computed the spin-singlet-triplet mixing angles $\bar{\gamma}_{J}$ : these are the fundamental dynamical quantities driving $\Delta A$. Our $\bar{\gamma}_{J}$ computation is realistic as we have used the Reid soft-core potential to generate the distortions in the $N N$ wave function. We have computed the spin-singlet-triplet angles using three different sources of isospin violation: (1) $\rho$ - $\omega$ mixing with the amplitude fixed at its on-shell value, (2) off-shell $\rho$ - $\omega$ mixing plus omega- and rhomeson exchange, and (3) on-shell $\rho$ - $\omega$ mixing plus omega- and rho-meson exchange. The first case, used in the original estimates of $\Delta A$, represents a "baseline" value, as it fits the data. A CSB potential of this magnitude accounts for a substantial fraction of the measured value of $\Delta A$ at $183 \mathrm{MeV}$. The second case, which should be regarded as our best estimate, yields values for $\bar{\gamma}_{J}$ that are within $10 \%$ of those obtained with on-shell $\rho-\omega$ mixing, for the important partial waves. In contrast, case (3) results in a factor-of-two enhancement relative to the original calculation using on-shell $\rho-\omega$ mixing. Two important results thus emerge from the present work. First, we have found a new source of isospin violation, namely in the $N N \omega$ vertex, which can fill the role demanded by the data. Second, we have shown that insisting upon a $\rho-\omega$ mixing amplitude held constant at its on-shell value, after including the contribution from omega-meson exchange, results in a class IV potential too large to be consistent with the IUCF $\Delta A$ measurement.

\section{ACKNOWLEDGMENTS}

We thank V. Dmitrašinović and S.J. Pollock for fruitful discussions, A. Thomas for a helpful suggestion, and S. Capstick for useful conversations. This work was supported by the

DOE under Contracts Nos. DE-FG02-87ER40365 (S.G. and C.J.H.), DE-FC05-85ER250000 (J.P.), and DE-FG05-92ER40750 (J.P.). 


\section{REFERENCES}

[1] T. Goldman, J.A. Henderson, and A.W. Thomas, Few-Body Systems 12, 123 (1992); Mod. Phys. Lett. A7, 3037 (1992).

[2] J. Piekarewicz and A.G. Williams, Phys. Rev. C 47, R2462 (1993).

[3] T. Hatsuda, E.M. Henley, T. Meissner, and G. Krein, Phys. Rev. C 49, 452 (1994).

[4] G. Krein, A.W. Thomas, and A.G. Williams, Phys. Lett. B 317, 293 (1993).

[5] K.L. Mitchell, P.C. Tandy, C.D. Roberts, and R.T. Cahill, Phys. Lett. B 335, 282 (1994).

[6] H.B. O'Connell, B.C. Pearce, A.W. Thomas, and A.G. Williams, Phys. Lett. B 336, 1 (1994).

[7] K. Maltman, University of Adelaide preprint ADP-95-20/T179 and hep-ph/9504237.

[8] L.D. Knutson et al., Nucl. Phys. A508, 185c (1990); Phys. Rev. Lett. 66, 1410 (1991); S.E. Vigdor et al., Phys. Rev. C 46, 410 (1992).

[9] G.A. Miller, A.W. Thomas, and A.G. Williams, Phys. Rev. Lett. 56, 2567 (1986).

[10] A.G. Williams, A.W. Thomas, and G.A. Miller, Phys. Rev. C 36, 1956 (1987).

[11] G.A. Miller, B.M.K. Nefkens, and I. Šlaus, Phys. Rep. 194, 1 (1990).

[12] M.J. Iqbal and J.A. Niskanen, Phys. Lett. B 322, 7 (1994).

[13] G.A. Miller, University of Washington preprint DOE-ER-40427-09-N94 and nuclth/9406023; G.A. Miller and W.T.H. van Oers, University of Washington preprint DOE-ER-40427-17-N94 and nucl-th/9409013.

[14] H.B. O'Connell, B.C. Pearce, A.W. Thomas, and A.G. Williams, University of Adelaide preprint ADP-95-15/T176 and hep-ph/9503332.

[15] S. Gardner, C.J. Horowitz, and J. Piekarewicz, Florida State University preprint FSUSCRI-95-37 and nucl-th/9505001.

[16] B. Holzenkamp, K. Holinde, and A. W. Thomas, Phys. Lett. B195, 121 (1987).

[17] L.M. Barkov et.al., Nucl. Phys. B256, 365 (1985).

[18] C.-Y. Cheung, E.M. Henley, and G.A. Miller, Nucl. Phys. A348, 365 (1980).

[19] A. Gersten, Phys. Rev. C 24, 2174 (1981).

[20] E.M. Henley and G.A. Miller, in Mesons in Nuclei, eds. M. Rho and D.H. Wilkinson (North-Holland, Amsterdam 1979).

[21] V. Dmitrašinović and S.J. Pollock, U. of Colorado preprint UC/NPL-116 and hep$\mathrm{ph} / 9504414$.

[22] A. N. Mitra and M. Ross, Phys. Rev. 158, 1630 (1967).

[23] A.W. Thomas, R.P. Bickerstaff, and A. Gersten, Phys. Rev. D 24, 2539 (1981).

[24] E. M. Henley and Z. Zhang, Nucl. Phys. A472, 759 (1987).

[25] M.J. Iqbal and J.A. Niskanen, Phys. Rev. C 38, 2259 (1988).

[26] L. K. Morrison, Ann. Phys. 50, 6 (1968).

[27] C. Yakin, M. Halil, and E. Aksahin, Phys. Scr. 20, 36 (1979).

[28] R. Machleidt, K. Holinde, and Ch. Elster, Phys. Rep. 149, 1 (1987).

[29] R. Machleidt, in Advances in Nuclear Physics, v. 19, eds. J.W. Negele and E. Vogt (Plenum, New York, 1989).

[30] Y. Nambu, Phys. Rev. 106, 1366 (1957); J. J. Sakurai, Ann. Phys. 11, 1 (1960); M. GellMann and F. Zachariasen, Phys. Rev. 124, 953 (1961).

[31] Donald H. Perkins, Introduction to High Energy Physics, Addison Wesley (Menlo Park, California), 1987. 
[32] D. B. Lichtenberg, Phys. Rev. D 40, 3675 (1989).

[33] Sidney A. Coon and Michael D. Scadron, Phys. Rev. C 26, 562 (1982).

[34] S. A. Coon and R. C. Barrett, Phys. Rev. C 36, 2189 (1987).

[35] J. Piekarewicz, Phys. Rev. C 48, 1555 (1993).

[36] K. Maltman, Phys. Lett. B313, 203 (1993).

[37] Chuan-Tsung Chan, E. M. Henley, and Th. Meissner, Phys. Lett. B343, 7 (1995).

[38] L. Tiator, C. Bennhold, and S.S. Kamalov, nucl-th/9404013.

[39] J. D. Walecka, Nuovo Cimento 11, 821 (1959); F. J. Ernst, R. G. Sachs, and K. C. Wali, Phys. Rev. 119, 1105 (1960).

[40] R. K. Bhaduri, Models of the Nucleon, Addison Wesley (Redwood City, California), 1988.

[41] R.V. Reid, Ann. Phys. (N.Y.) 50, 411 (1968). 


\section{FIGURES}

FIG. 1. Charge-symmetry-breaking component of the $N N$ potential as a function of $q^{2}$ arising from on-shell $\rho-\omega$ mixing (solid line), off-shell $\rho-\omega$ mixing (dashed line), omega-meson exchange (dash-dotted line), and rho-meson exchange (dotted line).

FIG. 2. Charge-symmetry-breaking component of the $N N$ potential as a function of $r$ arising from on-shell $\rho$ - $\omega$ mixing (solid line), off-shell $\rho$ - $\omega$ mixing (dashed line), omega-meson exchange (dash-dotted line), and rho-meson exchange (dotted line).

FIG. 3. The integrand of the spin-singlet-triplet mixing angle $\bar{\gamma}_{1}$ at $183 \mathrm{MeV}$ for three different estimates of CSB: on-shell $\rho$ - $\omega$ mixing (solid line), off-shell $\rho-\omega$ mixing plus omega- and rho-meson exchange (dashed line), and on-shell $\rho-\omega$ mixing plus omega- and rho-meson exchange (dash-dotted line). The value of $\bar{\gamma}_{1}$ for each estimate, which is simply the area under the appropriate curve, appears in parentheses next to its label. 


\section{TABLES}

TABLE I. Quark model charges. The superscripts denote scalar, pseudoscalar, vector-isoscalar, and vector-isovector quark charges, respectively.

\begin{tabular}{lcccc}
\hline \hline & $g^{\mathrm{s}}$ & $g^{5}$ & $g^{+}$ & $g^{-}$ \\
\hline$u$ & $+1 / 3$ & $+1 / 5$ & $+1 / 3$ & +1 \\
$d$ & $+1 / 3$ & $-1 / 5$ & $+1 / 3$ & -1 \\
\hline \hline
\end{tabular}

TABLE II. Proton, neutron, isoscalar, and isovector meson-nucleon coupling constants.

\begin{tabular}{ccccccc}
\hline \hline & $g^{\sigma}$ & $g^{\pi}$ & $g^{\omega}$ & $f^{\omega}$ & $g^{\rho}$ & $f^{\rho}$ \\
\hline$p$ & +1 & +1 & +1 & 0 & +1 & +4 \\
$n$ & +1 & -1 & +1 & 0 & -1 & -4 \\
0 & +1 & $\frac{3}{10} \frac{\Delta m}{m}$ & +1 & 0 & 0 & $\frac{3}{2} \frac{\Delta m}{m}$ \\
1 & 0 & +1 & 0 & $\frac{5}{6} \frac{\Delta m}{m}$ & 1 & +4 \\
\hline \hline
\end{tabular}

TABLE III. Meson masses, coupling constants, tensor-to-vector ratio, and cutoff parameters of the Bonn B potential.

\begin{tabular}{ccccc}
\hline \hline Meson & Mass $(\mathrm{MeV})$ & $g^{2} / 4 \pi$ & $f / g$ & $\Lambda(\mathrm{MeV})$ \\
\hline$\pi$ & 138 & 14.21 & - & 1700 \\
$\eta$ & 549 & 2.25 & - & 1500 \\
$\rho$ & 769 & 0.42 & 6.1 & 1850 \\
$\omega$ & 783 & 11.13 & 0.0 & 1850 \\
\hline \hline
\end{tabular}

TABLE IV. Spin singlet-triplet mixing angles $\bar{\gamma}_{J}$ (in degrees) at a laboratory energy of $T_{\text {lab }}=183 \mathrm{MeV}$. The values in parenthesis use a form factor computed from the quark model (see text for details).

\begin{tabular}{lccc}
\hline \hline$J$ & $\left.\langle\rho|H| \omega\rangle\right|_{\text {on }}$ & $\left.\langle\rho|H| \omega\rangle\right|_{\text {off }}+(\omega-\rho)$ & $\left.\langle\rho|H| \omega\rangle\right|_{\text {on }}+(\omega-\rho)$ \\
\hline 1 & $3.41 \times 10^{-2}$ & $3.32 \times 10^{-2}\left(3.66 \times 10^{-2}\right)$ & $6.70 \times 10^{-2}\left(7.22 \times 10^{-2}\right)$ \\
2 & $4.51 \times 10^{-2}$ & $4.40 \times 10^{-2}\left(4.88 \times 10^{-2}\right)$ & $8.77 \times 10^{-2}\left(9.52 \times 10^{-2}\right)$ \\
3 & $3.77 \times 10^{-3}$ & $3.71 \times 10^{-3}\left(4.71 \times 10^{-3}\right)$ & $6.00 \times 10^{-3}\left(7.55 \times 10^{-3}\right)$ \\
4 & $8.04 \times 10^{-4}$ & $8.58 \times 10^{-4}\left(1.12 \times 10^{-3}\right)$ & $1.18 \times 10^{-3}\left(1.55 \times 10^{-3}\right)$ \\
\hline \hline
\end{tabular}




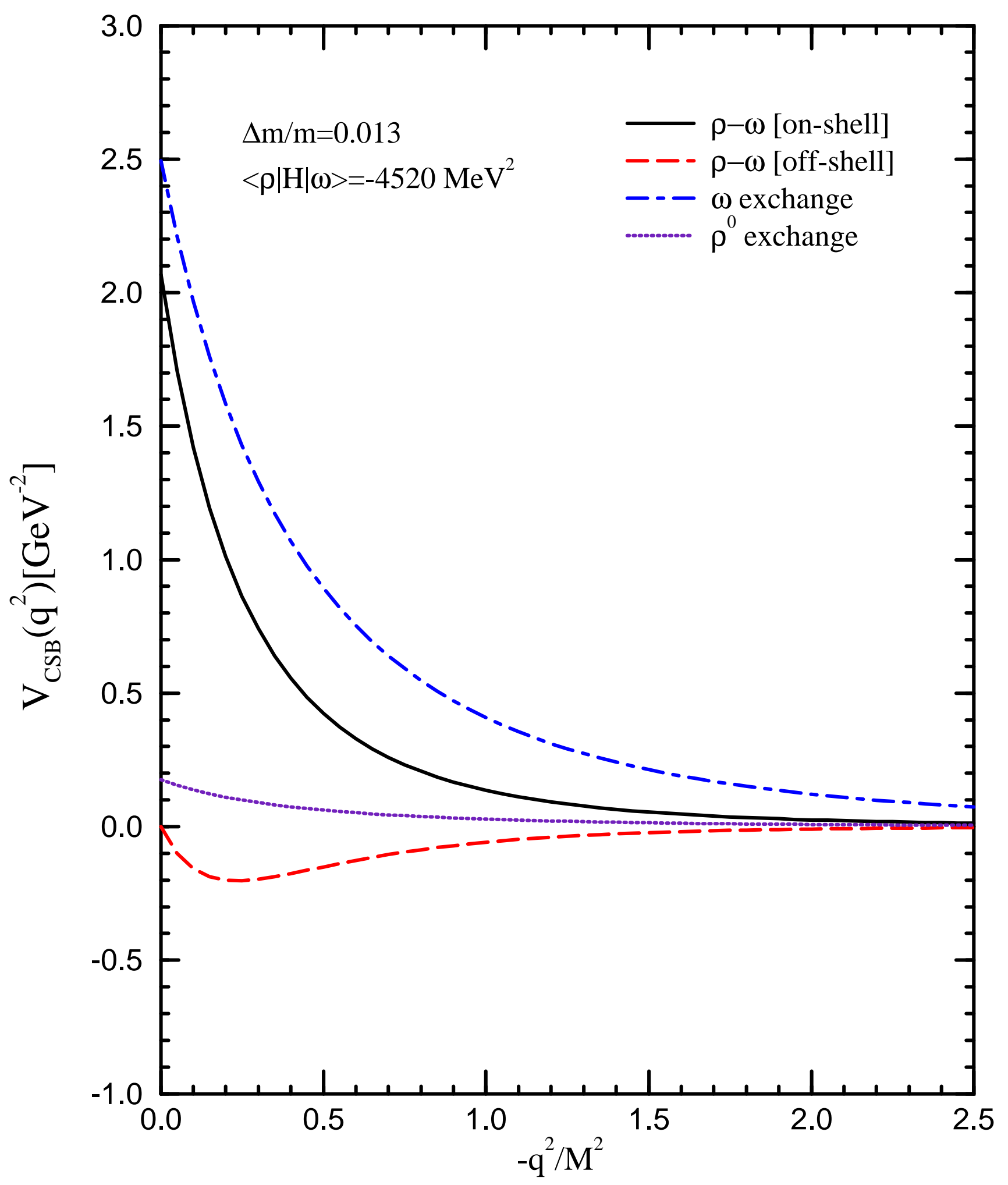




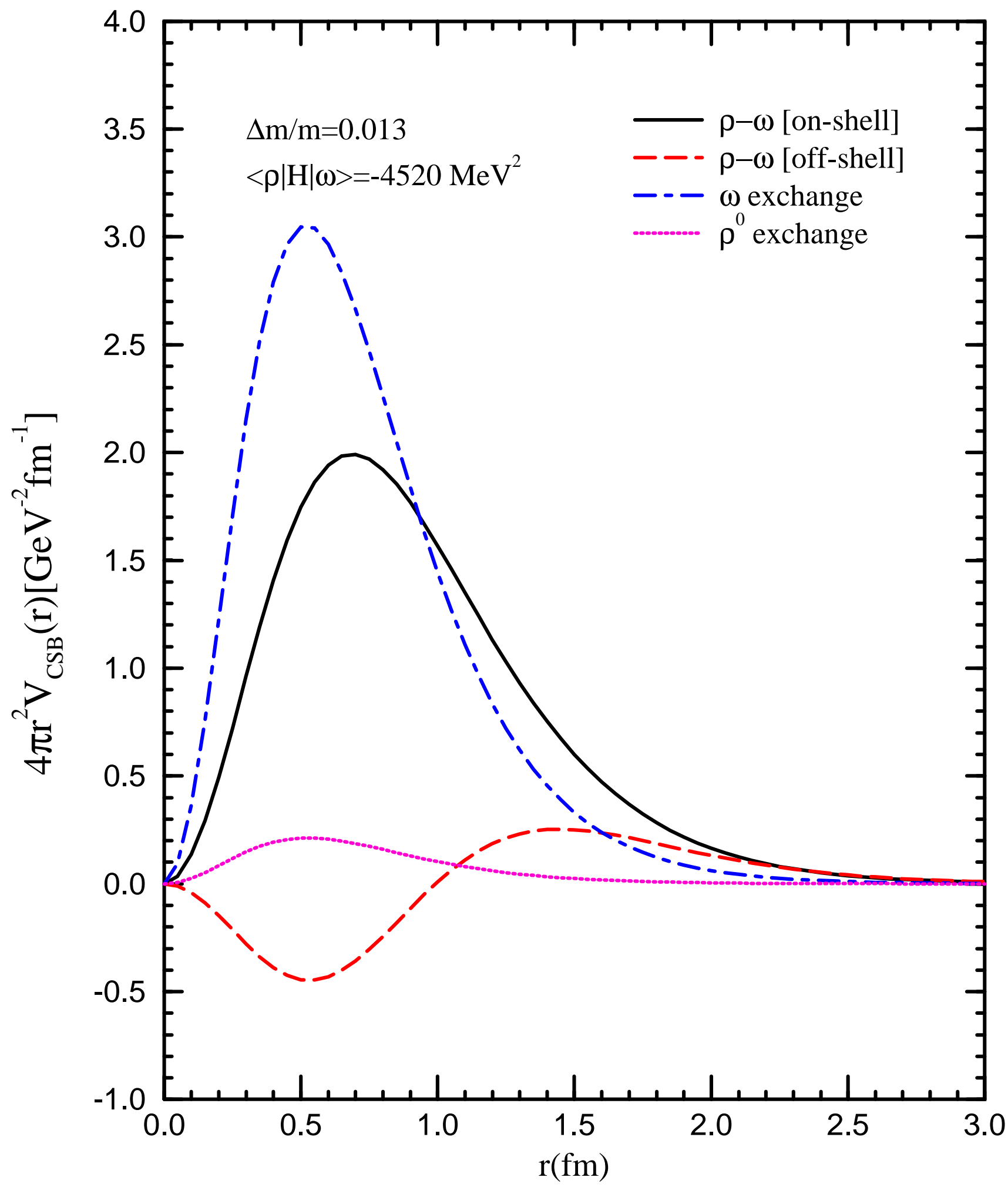




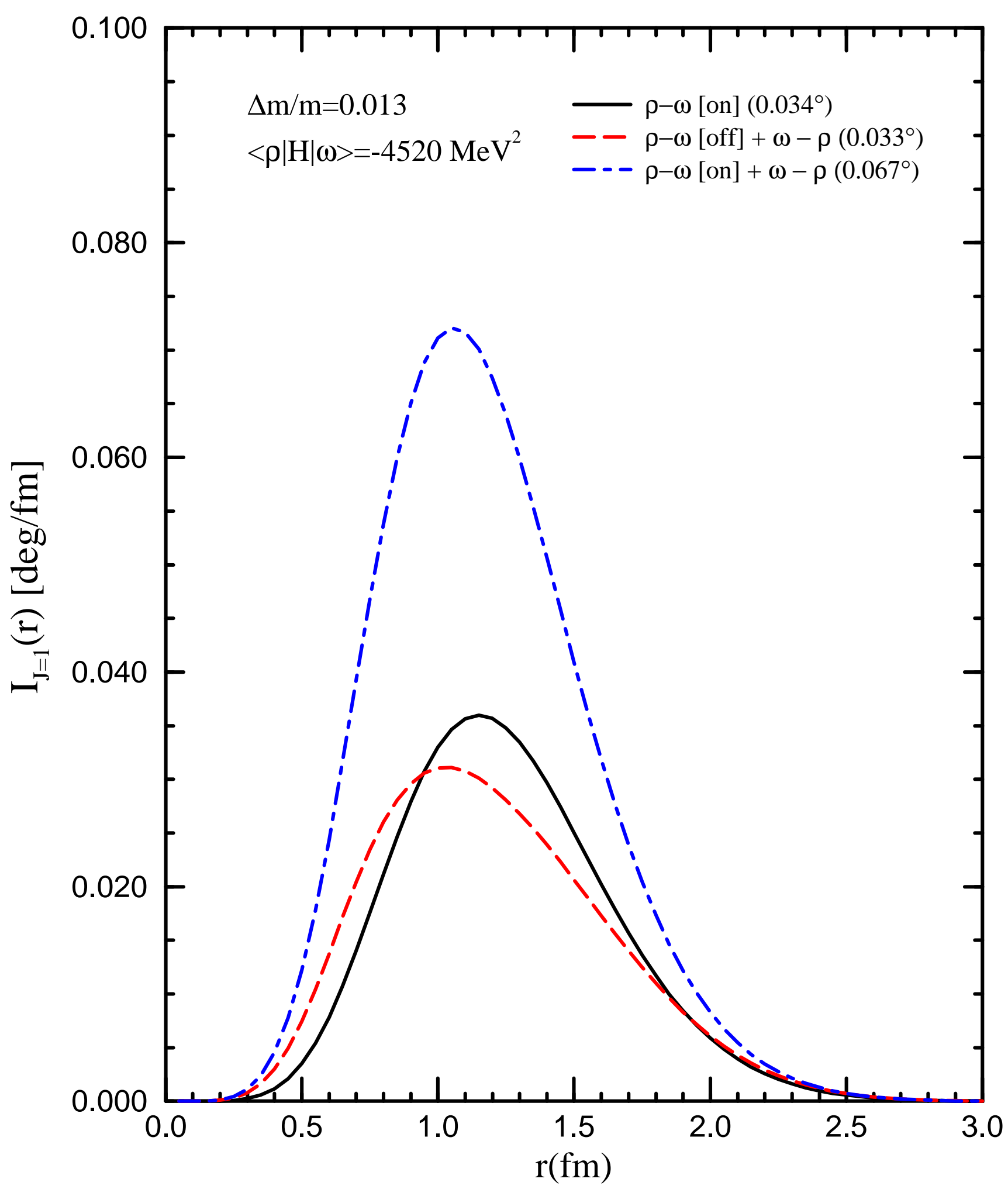

